\title{
LA PERSPECTIVE FONCTIONNELLE DE LA PHRASE ET L'ORDRE DES MOTS EN
}

\section{ALLEMAND}

\author{
Maria Paola TENCHINI \\ Université Catholique du Sacré Cœur de Brescia
}

\begin{abstract}
En): In this paper, elaborating on Daneš' idea of the "three-levels approach" to syntax, we advance the hypothesis that the global information structure of an utterance is the result of a synergistic action of semantic, syntactic and Theme-Rheme structures. In particular, we focus on the structural and formal factors of the functional sentence perspective, hence distinguishing between Theme-Rheme on the one hand and Given-New and PresuppositionAssertion on the other. The latter notions, though correlated to that of Theme-Rheme, are distinct from it. We apply this theoretical framework to the German language aiming at showing how, in this language, the linguistic means realizing the actual articulation in Theme and Rheme (particularly, word order and intonation) together with syntax and semantics determine the structure of an utterance and make it a 'communication unit' apt to be placed within specific verbal exchange contexts.
\end{abstract}

Keywords (En): functional sentence perspective; German word order; sentence structure levels; information structure

Mots clés (Fr): perspective fonctionnelle de la phrase; ordre de mots en allemand; niveaux de la structure énonciative; structure informationnelle

\section{Introduction}

Depuis MATHESIUS (1929), les Pragois ont toujours considéré le rapport entre articulation sémantique, articulation syntaxique et articulation actuelle de l'énoncé comme l'un des traits les plus caractéristiques de chaque langue. FIRBAS (1987 : 200), lors de son analyse du fonctionnement du dynamisme communicatif dans la perspective fonctionnelle de la phrase, a souligné que ce qui doit être considéré comme un caractère essentiel de la phrase, ce n'est pas la succession mais bien la coprésence (togetherness) des différents facteurs, soulevant ainsi la question de comprendre dans quelle mesure les fonctions exercées par un seul et même élément au niveau sémantique, syntaxique et fonctionnel coïncident.

En nous inspirant de l'idée de «three-levels approach » à la syntaxe de DANEŠ (1964), nous nous proposons de vérifier l'hypothèse selon laquelle la structure informative globale d'un énoncé est le résultat de l'action synergique de la structure sémantique, de la structure syntaxique et de la structure en Thème et Rhème. Notre intervention se concentrera en particulier sur les aspects de la perspective fonctionnelle de la phrase (PFP), dont la nature est à tous les égards structurale, avant d'être interprétative : en effet, le niveau de la manifestation linguistique est un niveau différent, et celui de l'interprétation cognitive est un niveau bien différent encore ${ }^{1}$. Par conséquent, les notions de Thème et de Rhème seront considérées comme bien distinctes de notions telles que le Donné et le

\footnotetext{
${ }^{1}$ Sur les quatre facteurs de la PFP soulignés par Firbas sur la base d'une pluripartition fonctionnelle de la phrase - soit la linéarité, la prosodie, la sémantique et le contexte - nous nous occupons donc en particulier des deux premiers (cf. FIRBAS, 1992).
} 
Nouveau ou le Présupposé et l'Asserté, bien qu'elles soient souvent mises en relation et considérées (de façon erronée) comme interchangeables d'un point de vue théorique.

Ce cadre théorique sera appliqué à la langue allemande : notre but est de montrer comment, en allemand, la syntaxe, la sémantique et l'articulation en Thème et en Rhème d'un énoncé (à travers les outils linguistiques qui la réalisent) contribuent à en déterminer la structure et à le faire devenir une «unité communicative » pouvant s'insérer dans le contexte d'un échange verbal.

\section{Les trois niveaux formels}

Prenons comme point de départ possible l'énoncé Charlotte küsst den Hauptmann (Charlotte embrasse le capitaine). Il traite d'un événement et des individus qui y participent : le baiser donné au capitaine par Charlotte.

Au niveau sémantique on peut le représenter de la façon suivante :

[EMBRASSER $(e) ;$ agent $(e)=$ Charlotte $;$ patient $(e)=$ le capitaine]

Dans cette phrase sont dénotés tous les événements ${ }^{2} e$ qui sont ceux d'embrasser et qui ont Charlotte comme Agent et le capitaine comme Patient. Agent et Patient sont deux des rôles sémantiques (Thêta) possibles que les individus peuvent avoir dans les états ou dans les événements.

Il s'agit du niveau de base, commun à toutes les langues, qui délimite un milieu structuré du monde dont l'énoncé dit quelque chose. Ce niveau est structuré seulement d'un point de vue ontologique et pas encore syntaxique; il ne présente pas encore d'articulation en Thème et Rhème, vu que celle-ci, comme nous le verrons, concerne les modalités par lesquelles une information est présentée, et non pas la structure du monde en tant que telle ${ }^{3}$.

Au niveau syntaxique, les langues disposent de règles spécifiques pour projeter la structure sémantique précédente (universelle) sur une structure syntaxique (particulière). D'habitude, le type d'événement $e$ est dénoté par le verbe, tandis que les individus sont dénotés par des syntagmes nominaux.

Un des passages fondamentaux de la structure sémantique à la structure syntaxique consiste dans la projection des rôles sémantiques (Thêta) sur les rôles syntaxiques. Dans notre cas, Charlotte est projetée sur le Sujet et le capitaine sur l'Objet. Ces opérations ont comme résultat des structures hiérarchiques morphosyntaxiques, dont une représentation très simplifiée pourrait être la suivante (elle n'est pas, bien sûr, la seule possible) :

\footnotetext{
${ }^{2}$ Nous utilisons «événements » au pluriel parce que «l'action d'embrasser le capitaine, effectuée par Charlotte» peut se référer également, au delà de l'événement singulier, à une pluralité d'événements (habitude ou itération). La restriction à un seul événement est contextuelle et n'appartient pas à la sémantique du verbe.

${ }^{3}$ Cette interprétation est donc différente de celle de SGall (1972), SGall (1982) et DaHL (1974) qui incorporent déjà l'articulation en Thème et Rhème à la représentation sémantique ou structure profonde.
} 


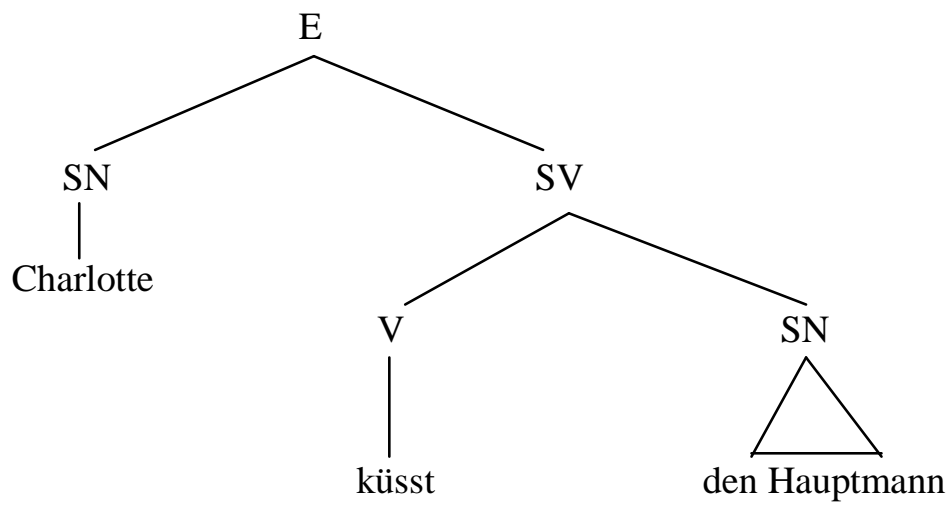

À ce niveau, l'ordre des constituants n'est pas marqué et toute forme intonative est encore absente.

Le troisième niveau, celui de la structure informative ou de la perspective fonctionnelle qui signale l'articulation d'un énoncé en Thème et Rhème, est obtenu à partir de la structure syntaxique de base à travers des mécanismes différents selon les langues, par exemple en allemand à travers des mouvements et l'accentuation de certains composants ${ }^{4}$.

Si nous ne changeons pas l'ordre des constituants de second niveau et si nous mettons l'accent sur la position par défaut, nous obtenons l'énoncé suivant :

Charlotte küsst den HAUPTMANN (les caractères en majuscule signalent une proéminence intonative).

Cependant, le même événement peut également être décrit par des énoncés différents de la forme de base précédente, différents de par l'ordre des constituants et la distribution de l'accent. Bien sûr, ils ne sont ni synonymes ni redondants parce que dans la réalité du discours ils ont des fonctions différentes, c'est-à-dire qu'ils sont adaptés (ou plus adaptés, ou mieux adaptables) à des contextes différents. Par exemple, si quelqu'un posait la question suivante :

Also, wie geht die Geschichte weiter, wer küsst den Hauptmann? (alors, comment l'histoire continue-t-elle ? Qui est-ce qui embrasse le capitaine ?)

on pourrait répondre:

Der Hauptmann wird von CHARLOTTE geküsst (Le capitaine est embrassé par CHARLOTTE)

CHARLOTTE, die küsst den Hauptmann (CHARLOTTE, c'est (bien) elle qui embrasse le capitaine)

Es ist CHARLOTTE, die den Hauptmann küsst (C'est CHARLOTTE qui embrasse le capitaine)

tandis que, dans le même contexte, les énoncés tels que :

Der HAUPTMANN wird von Charlotte geküsst (Le CAPITAINE est embrassé par Charlotte)

DER Hauptmann wird von Charlotte geküsst (C'est CE capitaine-là qui a été embrassé par Charlotte)

${ }^{4}$ «Zur Realisierung der Mitteilungsperspektive im Deutschen dienen vorrangig Intonation und Wortstellung, zusätzlich und subsidiär auch andere grammatisch-semantische Mittel (z.B. Artikel, hervorhebende Partikeln, Füllwort es usw.) » : BENEŠ (1971 : 164). 
Es ist der HAUPTMANN, der von Charlotte geküsst wird (C'est le CAPITAINE qui a été embrassé par Charlotte)

ne seraient pas très heureux d'un point de vue pragmatique.

Sans contexte, les énoncés structurellement et grammaticalement corrects sont en eux-mêmes tous admissibles, mais dans un contexte donné certains le sont, d'autres pas. Ceci ne signifie cependant pas que l'articulation d'un énoncé en Thème et en Rhème dépende du contexte ou qu'un contexte soit nécessaire pour la définir, autrement il n'existerait pas d'énoncés dont l'articulation effective soit inadaptée à un contexte. Si un énoncé est "plus adapté à certains contextes et moins à d'autres, cela ne prouve pas que le Thème et le Rhème dépendent du contexte, mais cela prouve exactement le contraire : la structure thématique de l'énoncé dépend de facteurs formels; ceux-ci étant établis, un énoncé peut être compatible ou non avec un contexte déterminé » (LOMBARDI VALLAURI, 2009 : 93, c'est nous qui traduisons).

En allemand, donc, un même événement $[\operatorname{KÜSSEN}(e)$; agent $(e)=$ Charlotte ; patient $(e)=$ der Hauptmann] peut constituer le point de départ de 'diverses' informations ; informations qui diffèrent selon si la structure syntaxique de base, qui dérive de la projection des rôles sémantiques sur les rôles syntaxiques, est rendue, au niveau informatif, à travers un ordre des mots déterminé par rapport à un autre ordre possible, à travers une certaine intonation, marquée ou non par rapport à celle existant par défaut, ou à travers des structures syntaxiques spécifiques de mise en évidence (voix passive, phrases scindées, dislocations). Ainsi, le troisième niveau est un niveau linguistique dans lequel les diverses structures linguistiques remplissent les différentes fonctions de mise en relief informatif nécessaires au discours.

\section{Thème et Rhème}

On a donné à Thème et à Rhème des dénominations et des définitions différentes, mais jamais parfaitement superposables. D'autres auteurs bien plus éminents ont souligné avant moi cette 'abondance' terminologique ${ }^{5}$ et ont essayé de la résoudre, mais à l'heure actuelle encore, dans des travaux tout à fait sérieux pas seulement sur l'allemand, on trouve un mélange terminologique plutôt trompeur. Ceci est principalement dû au fait que pour définir les concepts de Thème et Rhème on a eu recours - et aujourd'hui encore - aux catégories de la structure informative qui appartiennent à des plans différents (linguistique, cognitif, logique, textuel), sans les distinguer de façon convenable.

Les différentes définitions de Thème et de Rhème sont connues et amplement partagées, je ne m'attarderai donc pas à les rappeler en détail. J'aimerais cependant souligner ici qu'en premier lieu, Thème et Rhème doivent être distingués de Donné et de Nouveau et qu'en second lieu, ils doivent également être distingués de Présupposé et d'Asserté.

Donné et Nouveau, parce qu'il s'agit de notions cognitives, correspondent approximativement aux connaissances qui sont actives, semi-actives ou inactives dans la mémoire à court terme. En tant que tels, ils dépendent du contexte. Bien

\footnotetext{
${ }^{5}$ Cf. par ex. DANEŠ (1974), Dressler (1974), BENEš (1971) et LAMBrecht (1994, notamment le chapitre 2).
} 
que, dans la majeure partie des cas, le Thème soit plus adapté à véhiculer l'information la plus connue et le Rhème celle qui est nouvelle (cf. MATHESIUS, 1929 : 102), il n'est pas dit qu'il en soit toujours forcément ainsi. Par exemple, dans :

Ich habe Charlotte getroffen. Es war SIE, die den Hauptmann geküsst hat (J'ai rencontré Charlotte. C'est ELLE qui a embrassé le capitaine)

le Rhème du second énoncé est «sie », même si son référent a déjà été introduit dans l'énoncé précédent et est rappelé anaphoriquement.

Vice versa, même le Thème peut véhiculer une information nouvelle. Par exemple, en réponse à :

Warum hat Charlotte geschrien? (Pourquoi Charlotte a-t-elle crié ?)

dans l'énoncé suivant :

Während sie den Hauptmann küsste, hat sie sich EINEN ZAHN GEBROCHEN (Pendant qu'elle embrassait le capitaine, elle s'est CASSÉ UNE DENT)

$\mathrm{Si}$ cet énoncé est caractérisé par un contour intonatif unique, la phrase secondaire est le Thème et « einen Zahn gebrochen » est le Rhème, même si tous deux sont nouveaux d'un point de vue informatif par rapport à la question. Bien sûr, on peut également considérer comme valide la lecture selon laquelle c'est l'énoncé tout entier qui est le Rhème, mais de toute façon, ceci ne concerne pas la notion de nouveauté, mais plutôt celle d'adaptabilité au contexte. Pour cette raison, la structure Thème-Rhème doit toujours être tenue séparée des connaissances des locuteurs.

Thème et Rhème doivent également être distingués de Présupposé et Asserté qui divisent l'information sur la base de ce que le locuteur retient être une connaissance partagée ou non. Dans ce cas également, comme dans le précédent, il est vrai que le Thème est de préférence plus adapté à véhiculer des informations présupposées et le Rhème des informations assertées, cependant le contraire peut également être vrai. Un échange dans lequel c'est le Rhème qui véhicule le matériel présupposé peut être le suivant :

WEN hat Charlotte geküsst? (QUI est-ce que Charlotte a embrassé ?)

Sie hat den HAUTPMANN geküsst (Elle a embrassé le CAPITAINE)

dans lequel «den Hauptmann » est le Rhème même s'il présuppose l'existence de son référent (à travers l'article défini).

LOMBARDI VALLAURI (2009 : 102) affirme que «si la tournure est assertive mais ne fait pas partie du Rhème, son contenu est présenté comme une information accessoire par rapport au but communicatif » (c'est nous qui traduisons). Pour cette raison, dans :

Charlotte schläft UM DEN HAUPTMANN NICHT ZU KÜSSEN (Charlotte dort POUR NE PAS DEVOIR EMBRASSER LE CAPITAINE)

le contenu de la principale, même s'il est asserté, est présenté comme une information accessoire et c'est pourquoi il est Thème.

Donc Donné et Nouveau, Présupposé et Asserté concernent l'état cognitif des locuteurs ; Thème et Rhème concernent le matériel linguistique.

Ceci étant acquis, nous proposons la définition suivante: en tant que constituant du troisième niveau de la structure informative, le Rhème est la partie de la structure énonciative qui est mise en évidence à travers le matériel linguistique (en allemand, il s'agit principalement de l'ordre des mots et de la 
proéminence intonative) et qui sert, d'un point de vue fonctionnel, à souligner la partie de l'information considérée comme la plus importante. Tout ce qui n'est pas Rhème est Thème ${ }^{6}$, c'est-à-dire que le Thème est ce qui sert de fond ${ }^{7}$, de support à l'élément important d'un point de vue informatif, en fournissant des informations accessoires ou supplémentaires, mais pas indispensables à la détermination du but énonciatif.

Même si elles ne coïncident pas, cette définition a des points en commun avec celle, plus traditionnellement pragoise, de Thème entendu comme ce dont on parle, et de Rhème, entendu comme ce qui se dit à propos du Thème ; et ceci parce que les deux définitions se basent sur le rôle central de l'importance informative, sans tenir compte de notions comme celles de nouveauté ou de présupposition, et également parce que toutes deux considèrent le Thème et le Rhème comme des moments structuraux, quelles que soient les diverses manifestations dans les différentes langues. D'un côté, cette définition est cependant trop limitée parce qu'elle semble favoriser l'hypothèse selon laquelle le Thème serait à gauche, i.e. le premier élément de la phrase ${ }^{8}$, ce qui n'est pas une structure obligatoire, du moins dans les langues à ordre des mots 'libre'. D'un autre côté, cette définition est trop large, parce qu'elle ne fournit pas les critères sur lesquels se fonder pour délimiter ce dont on parle. En fait, dans le cadre des approches logiques à la sémantique, ce dont parle la phrase, ce sont ses arguments, et ce qu'elle dit est le prédicat; de plus, dans de nombreuses approches traditionnelles, le sujet est ce dont parle la phrase, tandis que le prédicat est ce que dit la phrase de ce sujet.

\section{L'ordre des mots et la perspective fonctionnelle de la phrase}

En allemand, l'ordre des mots est caractérisé autant par des restrictions précises que par une certaine 'liberté' dans la disposition. Les restrictions morphosyntaxiques, qui en allemand interviennent par exemple sur la position du verbe et sur la structure interne des syntagmes nominaux, sont déterminées par le second des niveaux définis plus haut, c'est-à-dire par la structure syntaxique de la langue. La liberté de disposition" contribue à manifester l'articulation de l'énoncé en Thème et en Rhème et a pour cette raison une fonction essentiellement pragmatique.

En réalité, au lieu de parler de succession libre ou de position obligée, il nous semblerait plus approprié de parler en général d'une flexibilité dans l'ordre des mots dont l'issue dépend fondamentalement du but communicatif. Beneš, dans ses

\footnotetext{
${ }^{6}$ Pour des raisons d'espace, nous ne tenons pas compte ici des différentes expansions de l'échelle de thématicité / rhématicité.

${ }^{7}$ Les études les plus récentes ont enrichi l'analyse de la structure informative de l'énoncé avec des notions telles que celles d'information(s) de premier plan (foregrounded discourse portions, Vordergrundsinformation) et d'information(s) d'arrière-plan (backgrounded discourse portions, Hintergrundsinformation). Cf. par ex. LAMBRECHT (1987) et ZIFONUN et al. (1997).

${ }^{8}$ Cette hypothèse est soutenue en ce qui concerne l'allemand par ex. par Boost (1955:26). La tripartition de BENEŠ (1971 : 164) en Basis ou Ansatz (élément que l'énoncé ou le discours prend comme point de départ), Thème et Rhème semblerait plus intéressante, mais en réalité, la notion de Ansatz est orthogonale par rapport à celle de Thème et Rhème.

${ }^{9}$ En allemand en effet, la flexion nominale contribue, mis à part les syncrétismes (mais le plan sémantique peut y pourvoir), à signaler le cas syntaxique des constituants nominaux.
} 
travaux sur l'allemand ${ }^{10}$, définissait l'ordre des mots de langues telles que l'allemand, comme sinngebunden (lié au sens) ou sinnbedingt (conditionné par le sens) (BENEŠ, 1967 : 24). D'ailleurs, dans un travail de 1962, Beneš encore a exemplifié une longue série de stratégies linguistiquement codifiées ayant pour but de contourner la rigidité de la norme syntaxique qui prévoit des positions fixes pour le verbe, en faveur de la perspective fonctionnelle ${ }^{11}$.

Cependant, en allemand, une analyse de l'énoncé sous une perspective fonctionnelle, ou communicative (Mitteilungsperspektive), ne peut ignorer que parmi les instruments linguistiques qui déterminent sa structure informative et communicative, tout comme l'ordre des mots, le rôle de la mise en relief prosodique est essentiel. Celle-ci est également caractérisée par une certaine 'liberté d'intervention' puisque l'allemand ne prévoit pas de position fixe pour l'accent de phrase, même s'il existe une position par défaut placée dans la partie terminale de l'énoncé, qui est d'ailleurs également la position du Rhème par défaut.

L'allemand est donc une langue dans laquelle l'ordre des mots et la prosodie ont le même niveau de flexibilité, contrairement à l'anglais par exemple, qui accorde un poids différent aux liens syntaxiques et aux liens prosodiques et rythmiques ${ }^{12}$.

L'ordre des mots et la prosodie peuvent coopérer ou bien, en cas de différence d'un point de vue fonctionnel, une des deux structures peut céder le pas à l'autre dans le signalement de l'information considérée comme la plus importante ${ }^{13}$.

La position du verbe (formes finies, formes infinitives et participes, y compris les préfixes séparables) sépare l'énoncé en trois champs topologiques principaux : Vorfeld, Mittelfeld et Nachfeld ${ }^{14}$ et forme ce que l'on appelle la verbale Klammer, c'est-à-dire la structure à parenthèses typique de l'allemand.

La possibilité d'effectuer, au moyen du déplacement de constituants au-delà des limites des champs principaux, des dislocations à gauche (Linksversetzungen) et des dislocations à droite (Rechtsversetzungen) caractérise deux autres segments de collocation, qui ne doivent pas nécessairement être occupés. La structure de la phrase complète peut être visualisée comme suit ${ }^{15}$ :

\footnotetext{
${ }^{10}$ Nous devons beaucoup à Beneš pour l'extrême clarté et la synthèse de ses études sur l'ordre des mots en allemand.

${ }^{11}$ Il suffit de rappeler ici, à titre d'exemple, le cas de ce qu'on appelle la «position initiale couverte » (gedeckte Anfangsstellung). Par ce terme, nous nous référons aux cas où, à cause de la perspective fonctionnelle, apparait une particule atone (en général es) en tête de la phrase déclarative qui exige le verbe fini en deuxième position; cette particule sert de «explétif », afin que le verbe apparaisse comme premier élément de la communication avec une fonction thématique, sans perdre sa seconde position grammaticale: Es kommt der Frühling, au lieu de der Fruhling kommt (le printemps arrive). Cf. BENEŠ (1962: 11).

${ }^{12}$ Cf. par ex. MATHESiUs (1941-42) et Mathesius (1947).

${ }^{13}$ En reprenant l'exemple de la note 10, comparons Der Frühling KOMMT, Der FRÜHLING kommt et Es kommt der FRÜHLING.

${ }^{14}$ Litt. pré-champ, champ du milieu, post-champ.

${ }^{15}$ Un exemple de dislocation à gauche (soulignée) dans le linkes Aussenfeld peut être donné cidessous : (cf. ZIFONUN et al., 1997 : 1578)
}

\begin{tabular}{|l|l|l|l|l|l|l|} 
Der Dichter, & der & kann & mit der Sprache praktisch & machen, & was er will & $x$
\end{tabular}

(littéralement : le poète, lui, peut faire avec la langue pratiquement ce qu'il veut). 


\begin{tabular}{|l|l|l|l|l|l|l|}
\hline $\begin{array}{l}\text { Linkes } \\
\text { Aussenfeld }\end{array}$ & Vorfeld & $\begin{array}{l}\text { Linke } \\
\text { Satzklammer }\end{array}$ & Mittelfeld & $\begin{array}{l}\text { Rechte } \\
\text { Satzklammer }\end{array}$ & Nachfeld & $\begin{array}{l}\text { Rechtes } \\
\text { Aussenfeld }\end{array}$ \\
\hline
\end{tabular}

ZIFONUN et al. (1997) signalent une structure à parenthèse de base secondaire ${ }^{16}$ qui concerne les éléments du Mittelfeld et qui dépend essentiellement de critères d'ordres syntaxiques et sémantiques, comme le fait d'être argument ou ajout du verbe, l'anaphoricité, le fait d'être animé ou non, le fait de jouer un certain rôle syntaxique et d'avoir un certain scope $e^{17}$.

Ces mêmes critères sont, de toute façon, valables pour l'énoncé en entier et contribuent à définir l'ordre de base «normal» (normale Wortstellung) dans lequel, par exemple, le Vorfeld est occupé par le sujet, de préférence s'il est animé, tandis que l'objet et les compléments se trouvent dans le Mittelfeld. Mais à coté de cette structure de base, d'autres structures sont également possibles et sont toujours admissibles d'un point de vue grammatical ; elles modifient parfois la séquence de base non marquée (non seulement du point de vue communicatif, mais également du point de vue structural) Thème-Rhème en Rhème-Thème.

Des études récentes sur l'ordre des mots en allemand (par exemple BADER, HÄUSSLER, 2010) confirment que l'ordre de base non marqué dépend des propriétés lexico-sémantiques des arguments du verbe et donc de la hiérarchie des rôles sémantiques ; elles reconnaissent également comme légitimes des déviations de cet ordre à des conditions informativo-structurales précises.

Cependant, si pour Zifonun et al. la structure informative se définit dans le Vorfeld et dans la position finale du Mittelfeld (ZIFONUN et al., 1997 : 1558), ce ne sont pas les seules positions de mise en relief : le linkes Aussenfeld, le rechtes Aussenfeld et le Nachfeld, quand il n'est pas occupé par les éléments qui l'occupent de façon préférentielle dans un ordre non marqué, peuvent également jouer ce rôle. La collocation des éléments dans ces secteurs-ci, unie à la proéminence intonative, contribue donc également à signaler l'information principale, ou l'une des informations saillantes et nécessaires à la transmission de la portée informative de l'énoncé.

Parfois, les positions de proéminence occupées dans une phrase peuvent être au nombre de deux (rarement plus), ou bien l'énonciation entière peut constituer le Rhème. Bien que la structure en Thème et Rhème soit déjà fournie au niveau formel, la lecture du Rhème en tant que Rhème élargi ou Rhème restreint (avec le sous-groupe du Rhème contrastif ${ }^{18}$ ), peut dépendre du contexte.

Nous proposons ci-dessous un exemple de dislocation à droite (soulignée) dans le rechtes Aussenfeld et interposée entre deux Nachfeld (gestern et ob das stimmt). Cf. ZIFONUN et al. (1997 : 1650).

\footnotetext{
\begin{tabular}{|l|l|l|l|l|l|l|l|}
$x$ & Ich & habe & sie & gefragt, & gestern, & die Monika, & ob das stimmt \\
\hline
\end{tabular}

(littéralement : je lui ai demandé hier, à Monica, si c'était vrai).

16 «Allerdings handelt es sich nicht um eine Klammer in dem Sinne, daß sich die beiden Teile - wie der der Satzklammer - gegenseitig bedingen» (ZIFONUN et al., 1997: 1557). Les éléments qui forment cette structure à parenthèse interne au Mittelfeld constituent pour ZIFONUN et al. (1997 : 1560) les principaux éléments de premier plan et d'arrière-plan (Hintergrunds- et Vordergrundseinheiten).

${ }^{17}$ Pour des schémas récapitulatifs, cf. ZifONUN et al. (1997 : 1558, 1560).

${ }^{18} \mathrm{Au}$ sujet de la notion de rhème contrastif, cf. par ex. CHAFE (1976) et DIK (1997: 326-338).
} 
Les éléments restants, c'est-à-dire les éléments thématiques, constituent l'information accessoire et leur disposition, même réciproque, est moins fonctionnelle, du point de vue du but informatif.

Afin de vérifier l'apport de nature structurale de l'ordre des mots et de l'intonation dans la détermination de l'articulation effective d'un énoncé, nous avons demandé à dix locuteurs natifs de signaler le ou les éventuels accents de proéminence présents dans certains énoncés et/ou textes écrits ${ }^{19}$.

Les exemples suivants sont extraits d'un seul microtexte (l'ordre original des phrases dans le texte était 1, 3,2). Ils sont composés de plus d'une unité intonative, chacune ayant un accent primaire et étant délimitée par des pauses.

En général, tous les informateurs ont mis en évidence deux positions de proéminence intonative, l'une perçue comme primaire (en majuscule) et l'autre perçue comme secondaire (texte souligné) : cette fois-ci cependant, le niveau de proéminence donné aux éléments mis en évidence et éventuellement leur extension a été différent. Les positions les plus largement admises sont le Vorfeld et la partie finale du Mittelfeld.

Dans le premier exemple, les éléments mis en évidence à travers la proéminence intonative ont été les suivantes :

1a) Die Anschläge von Oslo würden Norwegen VERÄNDERN, hat Ministerpräsident Stoltenberg gesagt, es werde ein Norwegen DAVOR und ein Norwegen DANACH geben.

(Les attentats d'Oslo TRANSFORMERONT la Norvège, a dit le Président Stoltenberg, il y aura une Norvège AVANT et une NORVÈGE APRÈS).

1b) Die ANSCHLÄGE VON OSLO würden Norwegen verändern, hat Ministerpräsident STOLTENBERG gesagt, es werde ein NORWEGEN DAVOR und ein NORWEGEN DANACH geben.

(Les ATTENTATS D'OSLO transformeront la Norvège, a dit le Président

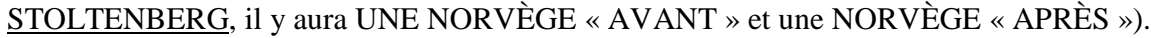

Quelques précisions: le nom du ministre-président a été marqué intonativement dans seulement deux cas (une fois par l'accent primaire et une autre par l'accent secondaire : la double possibilité est signalée par la police de caractère à la fois en majuscule et soulignée), faisant ainsi perdre à l'incise la fonction globale de Thème. L'élément établi dans le Vorfeld a été mis en évidence intonativement une seule fois, ce qui est probablement dû au fait que le Vorfeld n'est pas la position de défaut de la mise en relief, et encore moins quand il est occupé par le sujet. L'extension des éléments mis en évidence dans la troisième unité varie : $50 \%$ des informants a mis en évidence Norwegen davor et Norwegen danach, tandis que les autres ont mis en évidence seulement davor et danach, le référent étant considéré comme donné, et donc comme d'une importance mineure.

\footnotetext{
${ }^{19}$ La vérification a été effectuée en deux phases. Tout d'abord, les informants ont travaillé sur des phrases extraites de trois articles de journaux et présentées en désordre. Ensuite, certains d'entre eux ont travaillé sur les mêmes phrases présentées sous la forme du texte original, afin de vérifier si le cotexte peut influencer la lecture en terme de Thème et de Rhème. D'autres informants ont travaillé exclusivement sur les textes originaux complets. Pour des raisons d'espace nous ne reproduisons ici qu'un seul des trois microtextes. Nous désirons remercier les collègues, actuels et anciens, de l'Université Catholique de Brescia et de Milan qui ont participé au test.
} 
Dans le second exemple, la position initiale des deux unités a toujours été marquée intonativement (une lecture probablement préférée à cause du Rangattribut 'auch' précédant le pronom sujet). Les positions les plus partagées ont été :

2a) Auch WIR werden nicht mehr dieselben sein, auch wir KÖNNEN nicht mehr dieselben sein nach Oslo.

(NOUS non plus NOUS ne serons plus les mêmes, nous non plus ne POUVONS plus être les mêmes après Oslo)

2b) Auch WIR werden nicht mehr dieselben sein, auch wir können nicht mehr dieselben sein NACH Oslo.

(NOUS non plus NOUS ne serons plus les mêmes, nous non plus ne pouvons plus être les mêmes APRÈS Oslo)

En ce qui concerne la lecture de la seconde unité de 2) les éléments initiaux sont toujours accentués, même s'ils le sont de façon différente, tandis que dans deux cas seulement un élément qui se trouve dans le Nachfeld est mis en relief, après la limite syntaxique de l'énoncé ; celui-ci n'est pas la position par défaut de mise en relief mais est souvent utilisé par écrit dans ce but (cf. BENEŠ, 1968).

Le résultat de la lecture du troisième exemple a été double, parce que certains ont signalé deux proéminences intonatives, d'autres se sont limités à une seule :

3a) Noch sind nicht alle Hintergründe des Attentats GEKLÄRT, noch sind nicht alle Verbindungen des Attentäters ÜBERPRÜFT, aber SCHON JETZT lässt sich sagen: Auch für $\underline{U N S}$ in Europa bedeuten die Anschläge von Oslo eine Zäsur.

(Toutes les raisons de l'attentat n'ont pas encore été ÉCLAIRCIES, tous les contacts de l'auteur de faits n'ont pas encore été CONTRÔLÉS non plus, mais on peut DÈS MAINTENANT affirmer ceci : pour NOUS aussi en Europe, les attentats d'Oslo marquent une rupture)

3b) Noch sind nicht alle Hintergründe des Attentats GEKLÄRT, noch sind nicht alle Verbindungen des Attentäters ÜBERPRÜFT, aber schon jetzt lässt sich SAGEN: Auch für uns in Europa bedeuten die Anschläge von Oslo eine ZÄSUR.

(Toutes les raisons de l'attentat n'ont pas encore été ÉCLAIRCIES, tous les contacts de l'auteur de faits n'ont pas encore été CONTRÔLÉS non plus, mais on peut dès maintenant AFFIRMER ceci : pour nous aussi en Europe, les attentats d'Oslo marquent une RUPTURE)

Pour les informants qui ont produit 3a), les positions sélectionnées sont les mêmes; ce qui diffère parfois, c'est le niveau d'informativité primaire ou secondaire donné à travers la mise en relief intonative. En 3b), il semblerait que la position finale dans la phrase, qui par défaut indique le Rhème, influence significativement la lecture prosodique. Dans un cas seulement, toute la première phrase a été interprétée comme information de fond, i.e. comme Thème, et n'a pas été marquée intonativement.

Dans la lecture des phrases sous forme de texte, les informants ont marqué grosso modo les mêmes positions et la même proéminence intonative que dans les exemples 1) et 2), tandis qu'ils ont modifié la lecture de l'exemple 3), dans lequel ils ont parfois mis en évidence intonative les syntagmes nominaux également ${ }^{20}$.

\footnotetext{
${ }^{20}$ Les éléments de connexion (noch ... nicht répété deux fois, aber schon jetzt, auch für uns in Europa, auch wir, dieselben) sont mis en évidence par la proéminence intonative seulement dans un
} 


\section{Conclusion}

Le résultat le plus intéressant de ce test est, à notre avis, le fait qu'il montre que pour un ordre de mots donné, la prosodie tend à être uniforme, c'est-à-dire à mettre en relief rhématique tendanciellement les mêmes éléments. En principe, si dans chaque unité deux positions de proéminence intonative ont été définies, l'une perçue comme primaire et l'autre comme secondaire, celles-ci se trouvent préférentiellement dans la partie initiale et dans la partie finale de celle-ci. Il s'agit évidemment de positions de mise en relief qui sont dans un certain sens structurellement détérminées (Vorfeld, partie finale du Mittelfeld et éventuellement Nachfeld). En cas de proéminence intonative unique, celle-ci se trouve dans la position de défaut du Rhème.

Un des problèmes que cette enquête laisse ouvert est si, en cas de double proéminence, les éléments mis en relief constituent deux Rhèmes de même rang pour la structure informative, ou bien un Rhème principal et un secondaire ${ }^{21}$.

\section{BIBLIOGRAPHIE}

BADER Markus, HÄUSSLER Jana (2010), Word order in German: A corpus study, Lingua, 120, p. 717-762.

BENEŠ Eduard (1962), Die Verbstellung im Deutschen, von der Mitteilungsperspektive her betrachtet, Philologia Pragensia, V, 1, p. 6-19.

BENEŠ Eduard (1967), Die funktionale Satzperspektive (Thema-RhemaGliederung) im Deutschen, Deutsch als Fremdsprache, 4, p. 23-28.

BENEŠ Eduard (1968), Die Ausklammerung im Deutschen als grammatische Norm und als stilistischer Effekt, Muttersprache, 78, p. 289-298.

BENEŠ Eduard (1971), Die Besetzung der ersten Position im deutschen Aussagensatz, in Moser Hugo (éd., 1971), Fragen der strukturellen Syntax und der kontrastiven Grammatik, Düsseldorf, Schwann, p. 160-182.

Boost Karl (1955), Neue Untersuchungen zum Wesen und zur Struktur des deutschen Satzes : der Satz als Spannungsfeld, Berlin, Akademie-Verlag.

CHAFE Wallace L. (1976) Giveness, contrastiveness, definiteness, subjects, topics and point of view, in: Li Charles N. (éd., 1976), Subject and topic, NewYork/London, Academic Press, p. 25-55.

DAHL Östen (1974), Topic-comment structure in a generative grammar with a semantic base, in: DANEŠ František (éd., 1974), p. 75-80.

DANEŠ František (1964), A three level approach to syntax, in: Travaux Linguistiques de Prague 1, Praha, Academia, p. 225-240.

DANEŠ František (éd., 1974), Papers on functional sentence perspective, Praha, Academia.

DANEŠ František (1974), Zur Terminologie der FSP, in: DANEŠ František (éd., 1974), p. 217-222.

cas, en 3). Ceci est probablement dû au fait que, étant donné le contexte, les constituants référentiels sont encore actifs dans la mémoire à court terme et sont considérés comme donnés.

${ }^{21}$ À ce sujet, il serait intéressant d'établir une comparaison avec le finlandais, langue dans laquelle la présence, dans une phrase simple, de deux Rhèmes est possible, l'un placé en position initiale et l'autre en position finale. Cf. SORNICOLA (2006 : 394). 
DIK Simon C. (1997), The Theory of Functional Grammar. Part 1: The Structure of the Clause, Berlin/New York, De Gruyter.

DRESSLER Wolfgang (1974), Funktionelle Satzperspektive und Texttheorie, in: DANEŠ František (éd., 1974), p. 87-105.

FIRBAS Jan (1987), On the Operation of Communicative Dynamism in Functional Sentence Perspective, Leuvense Bijdragen, 76, p. 289-304; trad. it. Il funzionamento del dinamismo comunicativo nella prospettiva funzionale della frase, in: Sornicola R., SvobodA A. (éd., 1991), p. 195-209.

FIRBAS Jan (1992), Functional Sentence Perspective in Written and Spoken Communication, Cambridge, Cambridge University Press.

LAMBRECHT Knud (1987), On the status of SVO sentences in French discourse, in: TOMLIN Russell (éd., 1987), Coherence and grounding in discourse, Amsterdam, John Benjamins, p. 217-262.

LAMBRECHT Knud (1994), Information structure and sentence form. Topic, focus, and the mental representations of discourse referents, Cambridge, Cambridge University Press.

LOMBARDI VALLAURI Edoardo (2009), La struttura informativa. Forma e funzione degli enunciati linguistici, Roma, Carocci.

MATHESIUS Vilém (1929), Funkční lingvistika, in: Sborník přednášek proslovených na Prvém sjezdu čsl. profesorů filosofie, filologie a historie v Praze 3. - 7. dubna 1929, Praha, Stálý přípravný výbor sjezdový, p. 118130 ; trad. it. La linguistica funzionale, in: SoRniCOLA R., SVOBODA A. (éd., 1991), p. 97-112.

MATHESIUS Vilém (1941-42), Ze srovnávacích studií slovosledných, Časopis pro moderní filologii, 28, p. 181-190; 302-307, trad. it. Sugli studi comparativi sull'ordine delle parole, in: SoRNICOLA R., SvOBODA A. (éd., 1991), p. 319345.

MATHESIUS Vilém (1947), O funkci podmětu, Čeština a obecný jazykozpyt, p. 277-285, trad. it. Sulla funzione del soggetto, in: SoRNICOLA R., SvOBODA A. (éd., 1991), p. 148-158.

SGALL Petr (1972), Topic, Focus and the Ordering of Elements in Semantic Representations, Philologica Pragensia, 15, p. 1-14, trad. it. Topic, fuoco e ordine degli elementi nelle rappresentazioni semantiche, in: SORNICOLA R., SvobodA A. (éd., 1991), p. 259-284.

SGALL Petr (1982), Wortfolge und Fokus im Deutschen, in: ABRAHAM Werner (éd., 1982), Satzglieder im Deutschen, Tübingen, Narr, p. 59-74.

SORNICOLA Rosanna, SvoBODA Aleš (éd., 1991), Il campo di tensione. La sintassi della scuola di Praga, Napoli, Liguori.

SORNICOLA Rosanna (2006), Interaction of syntactic and pragmatic factors on basic word order in the languages of Europe, in: Bernini Giuliano, Schwartz Marcia L. (éd., 2006), Pragmatic Organization of Discourse in the languages of Europe, Berlin/New York, De Gruyter, p. 357-544.

ZIFONUN, Gisela, HoFFMANN, Ludger, STRECKER, Bruno et al. (1997), Grammatik der deutschen Sprache, Berlin, New York, de Gruyter. 